\title{
Multicasting Based Enhanced Proactive SOURCE ROUTING IN MANETS
}

\author{
${ }^{1}$ Deepa Dasarathan and ${ }^{2}$ P. Nirmal Kumar \\ ${ }^{1}$ Research Scholar, Department of Electronics \& Communication Engineering, \\ ${ }^{1}$ Sathyabama University, Chennai, India \\ ${ }^{2}$ Associate Professor, Department of Electronics \& Communication Engineering, \\ ${ }^{2}$ College of Engineering, Guindy, Anna University, Chennai, India
}

\begin{abstract}
:
Mobile Ad-hoc Network (MANET) is an accumulation of movable nodes organizing a irregular topology without centralized administration. In a MANET, multicasting is a significant technique for utilizing data communication system. Multicasting based enhanced proactive source routing is proposed in this paper for Mobile Ad hoc Networks. It explains an innovative multicasting algorithm that considers the transmission energy and residual energy while forwarding the data packets. It improves the network throughput and raises the network lifetimes. Simulation analysis is carried in this proposed system and this method shows improved performance over the existing system.
\end{abstract}

\section{KEYWORDS:}

Mobile Ad hoc Network, residual energy, transmission energy, simulation analysis.

\section{INTRODUCTION}

Mobile Ad hoc Network (MANET) is a multi-hop wireless network that is composed of mobile hosts communicating with each other through wireless links. MANET is used in many practical applications, including personal area networks, military environments, home area networking and search a rescue operations.

A MANET consists of nodes acting as host and router in a wireless infrastructure-less communicating network. The nodes communicate with one other over a wireless link with each node acting as host and router. Because of mobility of node the topology of the network changes, thus routing becomes crucial. Sufficient guarantee of Quality of Service (QoS) is required for delivery of information.

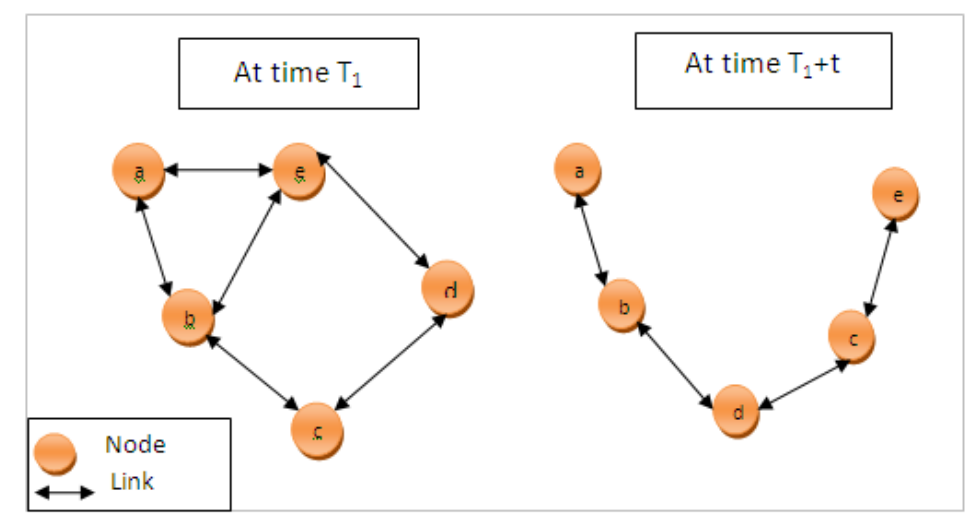

Figure.1 Working of a MANET

DOI: $10.5121 / \mathrm{ijcnc} .2017 .9607$ 
Figure. 1 shows the topology modified based on node mobility in MANET. At time T1 the nodes are formed in one structure and the time $\mathrm{T} 1+\mathrm{t}$ the nodes have changed another structure. Thus every time the node changes connectivity in MANET.

Multicast routing is one of the vital aspects in wireless ad-hoc networks. In multicast routing, the messages are sent to multiple specified destination nodes from a single source through utilizing multiple intermediate forwarding relay nodes [1]. This routing technique reduces transmission overhead, control message overhead, power consumption and network partitioning. In the field of wireless ad-hoc networks, geographic-based routing schemes have become important due to their localized operation, reduced computation and storage overhead and scalability with the required number of nodes. Proactive Source Routing (PSR) to help opportunistic data transmission in MANETs. In PSR, every node keeps a breadth-first search spanning tree of the network rooted at it. This data is sporadically substituted between neighbouring nodes for efficient network information. Proactive Routing refers to the ready availability of routes while there is data to be sent from one end to another.The PSR routing used to reduce the routing overhead and make network efficiency in MANET.

Our contributions in this paper can be summarized as follows:

1) We explain a Multicasting based Enhanced Proactive Source Routing (MEPSR) protocol for WSNs.

2) Multicasting is an important task because it reduces the data transmission duration and transmission costs for an application compared to the unicast transmission.

3) The route node selection based on Confidential Energy. The Confidential Energy is computed by remaining energy and Environmental standard energy of every node.

4) Here, Cost-aware based routing scheme can be useful to address the data delivery necessities.

The continuation of this paper is structured as follows. Section II reviews related work on routing protocol in MANETs. Section III presents details description of Multicasting based Enhanced Proactive Source Routing in MANETs. In section IV simulation analysis and results are discussed. Section V concludes this paper.

\section{RELATED WORKS}

Power Aware multicast algorithm [1] for extends the lifetime of the node and network without degrading the throughput. Energy Balanced Routing Method (EBRM) [3] based on Forward Aware Factor (FAF). In this scheme, the relay node selection based on knowledge of link weight and forward energy density. The forward communication area computes forward energy density that constitutes by link weight, and energy-balance routing. Thus it provides prolongs the function lifetime and balances the energy consumption. To avoid the energy consumption caused by the inside attack initiated by the malicious nodes, an Energy Efficiency routing with Node Compromised resistance (EENC) based on Ant Colony Optimization was developed [4].

Trust-based data aggregation protocol [5] reduces the nodal energy consumption and extends the life of the networks. Efficient Power Aware Routing protocol (EPAR) [2] for improve the network reliability based on data transmission power, capacity of node. The energy-aware routing algorithms for reduce the energy utilization in the routing path.

Vague set measurement technique [6] for improve energy efficient route in a network. Vague set measurement technique mainly used interval-based membership where each parameter such as 
energy and distance is used as an element of vague set. Adaptive HELLO message [11] for determines the local link connectivity information among nodes to reduce the energy consumption of mobile nodes. Energy efficient routing protocol network efficient clustering [12] along with location management scheme for reduces the re-clustering delay, lower the energy consumption and network life time.

Residual Energy based Reliable Multicast Routing Protocol (RERMR) [7] for increased packet delivery and forwarding rate and network lifetime. In this scheme stability depend on node familiarity and trustable based route is formed. Energy-Efficient Inter-Domain Routing Protocol [10] for improved the energy efficiency with low overhead. It select the route based on clustering techniques, virtual coordinates, Ant Colony Optimization. In this scheme, the data packet is sent from a source to a destination via internal and external connected gateways in different domains. The inter-domain routing based on bees' communication to handle a dynamic topology.

Energy Efficient Neighbor Coverage Protocol (EENCP) [8] for reduces to forward unnecessary RREQ in the network. EENCP establish the routing discovery based on node density and energy efficiency in a network. Thus it avoids both the redundant and needless data transmission. Efficient Packet Transmission and Energy Optimization scheme [9] for identified the networking gaps in a network. In this scheme, the $\mathrm{CH}$ (Cluster Head) shared the data to dual $\mathrm{CHs}$ that reduces the energy consumption. Also, it reduces the network congestion, interference and collisions. Adaptive Transmission Power scheme [13] for reduce the transmission power of control packets in the network.

Directional transmission based energy efficient routing protocol contains Power Efficient Gathering Sensor Information System (PEGASIS) and DSR routing protocols [14]. The hybridization of Genetic Algorithm (GA) and Bacterial Foraging Optimization (BFO) to identifies energy efficient optimal paths. Energy Efficient design of linear processing strategy [16] for improves the battery life and maximizing the energy efficiency. QoS aware routing [17] chooses the path based on bandwidth, nominal search, distance, and traffic condition. In this scheme the stable route selection according to Received Signal Strength Indication and QoS. It discover link break at the same instant of time and repair routes for all affected data flow. Energyaware Topology Control scheme [18] for minimizes the total transmission power desired and construct a topology that satisfy the QoS necessities among transmitter and receiver.

Stable Energy efficient QoS based Congestion and Delay aware Routing (SEQCDR) Protocol [15] utilized multiple metrics such as signal strength; queue length, drain rate and the delay for enhance the system performance. This scheme reduces the frequent link failures and packet losses of the network. SEQCDR provides a stable path among the sender and receiver, reduce the network delay and effective load balancing.

Efficient link failure strategy [19] for reduces the route failure in a network. It estimates the route break up based on link expiration metric and signal intensity level. QOS enabled fault-tolerant routing [20] recognize applicable route that used to select the alternate path while route failure. Backup route scheme [21] is established for improved the QoS routing. The possible failures of nodes and network are identified and start the backup routing. A path evaluation function is determined based on congestion, interference and energy drain rate are evaluated.

\section{Multicasting Based Enhanced Proactive Source Routing}

Multicasting based Enhanced Proactive Source Routing (MEPSR) in MANETs is proposed in this paper. The main goal of MEPSR technique is to increase the network lifetime and improve the packet delivery rate in MANET. 


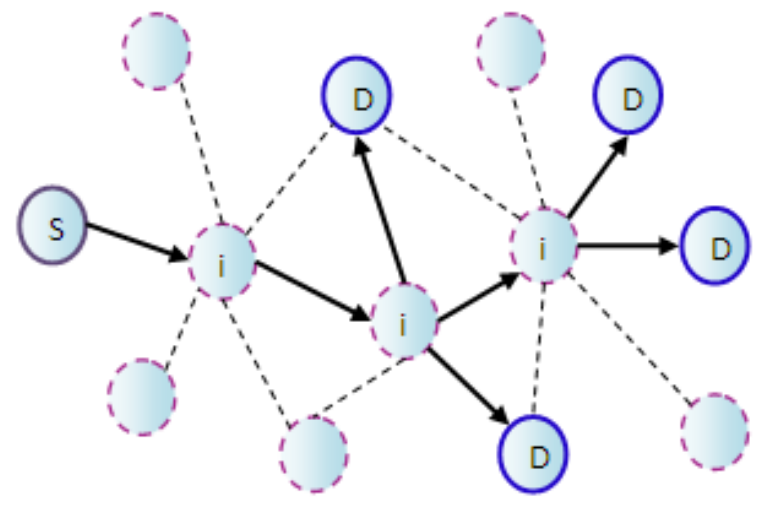

Figure 2 Example scenario of MEPSR Scheme

Figure 2 demonstrate that the example scenario of MEPSR scheme. Here, the single source transmits the multiple destinations known as multicasting. Where $S$ represents the Source, $D$ represents the Destination and $i$ represent the intermediate node.

\subsection{ROUTE DISCOVERY}

In the MEPSR, it employs energy aware optimal node-disjoint multipath from sender node to destination node by optimizing routing overhead. Two novel techniques were proposed to optimize the routing overhead. First novel technique is broadcast with low overhead approach; the main objective of this approach is to optimize the flooding of packets during route discovery. In this approach, during flooding of Route Request (RREQ) packets from sender to destination, many duplicate RREQ packets are eliminate by each intermediate node itself by using path's cost. Second novel approach is Computation of optimal and feasible energy aware node-disjoint multipath is done by destination node. In the MEPSR, The destination sends back multiple Rout Reply (RREP) packets to sender. Each RREP packet carries the path and its cumulative cost and confidential energy. The cumulative cost represents the node connectivity. In the traditional energy aware multipath routing, the sender node computes energy aware multipath from itself to destination during route discovery. If the destination sends all energy aware multiple paths in the RREP packets to sender via intermediate node then overhead is increased from destination to sender. In the MEPSR eliminates some unnecessary multiple paths that have less connectivity range in the route reply. The computation of the energy aware node-disjoint optimal paths and feasible are allocated to the destination node as an alternative of the sender node. It computes those paths based cost and sends back to sender node.

The sender consumes the energy communicates the message $(\mathrm{m})$ is determined by the equation 1 .

$$
E_{T r}(m, d)=E_{T r_{-} e l e c}(m)+E_{T r_{-} \text {amp }}(m, d)
$$

The energy consumption of receiver can be calculated by the equation 2 .

$$
\begin{aligned}
& E_{R}(m)=E_{R_{-} \text {elec }}(m) \\
& E_{R}(m)=m E_{\text {elec }}
\end{aligned}
$$


Where

$E_{\text {elec }} \rightarrow$ Energy consumption rate for communicate the 1 bit message

$\mathrm{m} \rightarrow$ Message

$\mathrm{d} \rightarrow$ Distance

The Confidential Energy is computed depend on the remaining energy and Environmental standard energy of each mobile node. Environmental standard Energy of node is evaluated by following formula.

$E S E=\frac{\sum_{j=1}^{h} R E_{j}}{h}$

$R E_{j} \rightarrow$ Residual Energy

$h \rightarrow$ Neighbor Nodes count

The Expectation Energy is received from the formula (4) below.

$E E_{j}=p \frac{E_{j}}{E S E}$

$p \rightarrow$ Desired percentage of mobile node

The mobile node confidence level of energy is measured by formula (5) below.

$C E=\frac{\frac{\sum_{j=1}^{h} R E_{j}}{h}+p \frac{E_{j}}{E S E}}{2}$

\subsection{ROUTE UTILIZATION}

The sender node categorizes received three paths based on the cost and stores it in to the cache as primary path, secondary path and ternary path. The sender node first selects primary path for data transmission to destination if it fails then next it selects secondary path for data transmission to destination if it also fails, then finally sender selects ternary path for data transmission to destination. If it also fails it invoke new discovery to find new energy aware node-disjoint path to destination. 


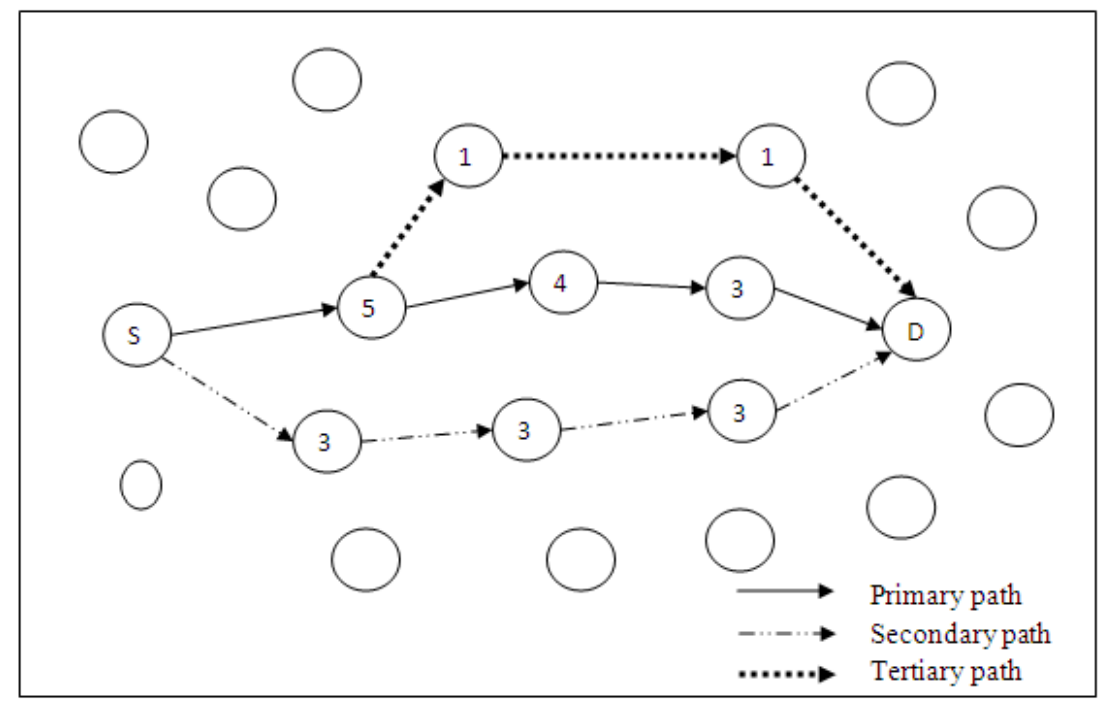

Figure 3: Steps in different path

Three types of paths exist in this criterion: primary path, secondary path and tertiary path and is shown in figure 3 . The primary path is considered as the basic path and is the efficient path for data communication. This path has the highest priority in data transmission. Next to primary path, secondary path takes the role of finding the efficient data. Tertiary path is the final path used for data transmission. This path selection technique has its role in selecting the best efficient of all the paths. Each path has its cost function and all the node with highest cost is selected as the primary path and so on. The cost is determined by the number of neighbors in the communication network.

\subsection{ROUTE MAINTENANCE}

The route maintenance in the MEPSR is same the route maintenance in the PSR. If currently using route is broken, then intermediate node sends a Route Error (RERR) packet to sender node to inform the sender node about the broken link. After receiving the RERR, the sender node removes that broken route from its cache and it uses the alternative route to destination if it is available otherwise it invokes new route discovery to find new routes to destination.

\section{Simulation ReSUlts}

The performance of the MEPSR is evaluated by using the Network simulator (NS2). The performance of the proposed scheme is evaluated by the parameters packet delivery ratio, packet loss ratio, average delay, throughput and residual energy. 
International Journal of Computer Networks \& Communications (IJCNC) Vol.9, No.6, November 2017

Table 1: Simulation parameters

\begin{tabular}{|l|l|}
\hline Parameter & Value \\
\hline Channel Type & Wireless Channel \\
\hline Simulation Time & $50 \mathrm{~ms}$ \\
\hline Number of nodes & 50 \\
\hline MAC type & 802.11 \\
\hline Simulation Area & $800 \times 800$ \\
\hline Transmission range & $230 \mathrm{~m}$ \\
\hline Mobility Model & Random Way Point \\
\hline Traffic Model & Constant Bit Rate \\
\hline Communication Protocol & User Datagram Protocol \\
\hline Antenna & Omini Antenna \\
\hline
\end{tabular}

\subsection{PACKet DeliVery RATe:}

Packet Delivery Rate is defined as the rate of packets delivered to the destination node. The PDR is calculated by the equation 3 .

$$
P D R=\sum_{0}^{n} \frac{\text { PacketsDelv }}{\text { Time }}
$$

Where

$\mathrm{n}=$ number of nodes. 
International Journal of Computer Networks \& Communications (IJCNC) Vol.9, No.6, November 2017

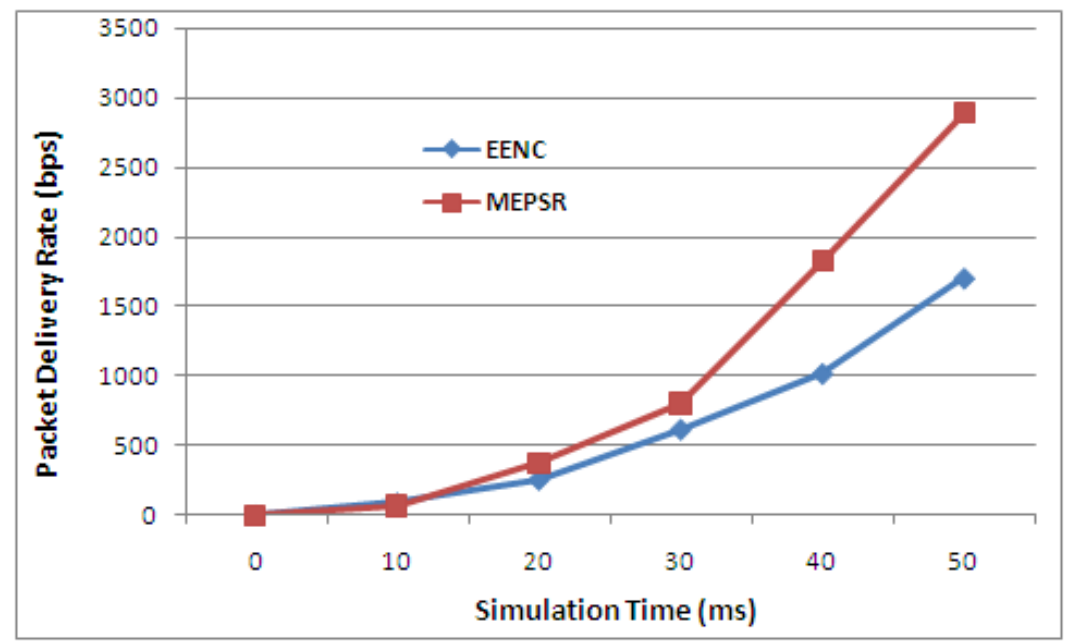

Figure.4 Packet delivery Rate

The figure 4 shows the PDR of the proposed scheme MEPSR is higher than the PDR of the existing method EENC. The delivery rate for MEPSR is constantly increasing as the simulation time is getting increased. The increase in the number of packets demonstrates the improvement in the delivery rate from source to destination. The MEPSR increase the node residual energy thus it improve the packet delivery from source to multiple destination.

\subsection{PACKet Loss Rate:}

Packet Loss Rate is defined as the number of packets lost per unit time. The formula used to calculate the PLR is given in equation 4.

$$
P L R=\sum_{0}^{n} \frac{\text { PacketsLost }}{\text { Time }}
$$

Where

$\mathrm{n}=$ number of nodes

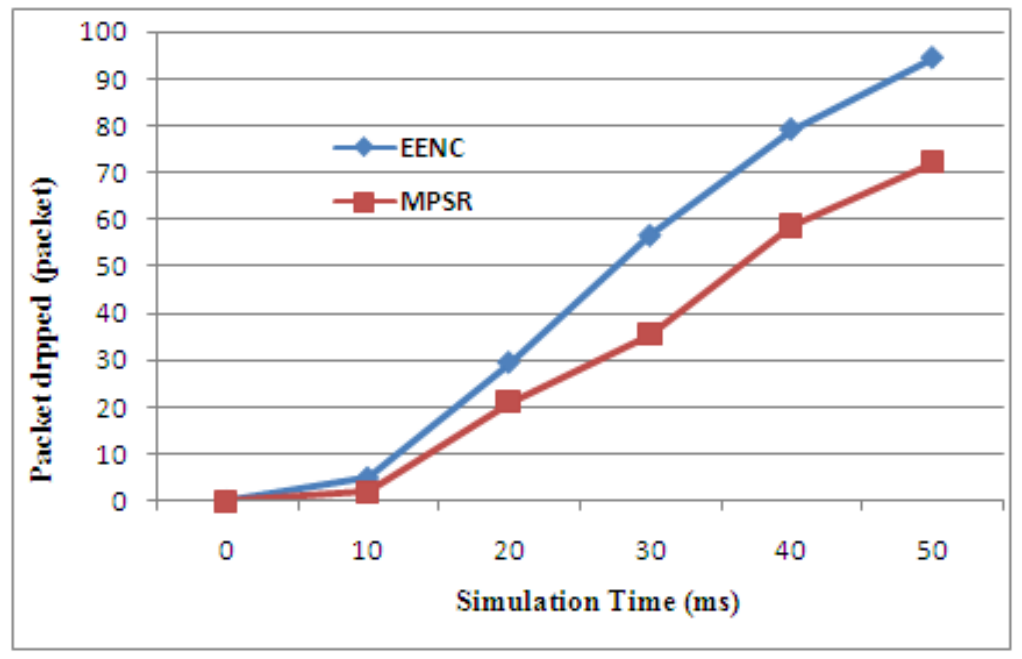

Figure.5 Packet Loss Rate 
The PLR of the proposed scheme MEPSR is lower than the existing scheme EENC in Figure 5. Lower the PLR indicates the higher performance of the network. As long as there is decrease in packet loss, the information sent from source will reach the destination successfully. The quality of the data will be automatically improved in this term of regard. The performance of the network will therefore be automatically improved.

\subsection{AVERage Delay:}

Average Delay is defined as the time difference between the received and sent packets to the total number of nodes. It is measured by the equation 5 .

Average Delay $=\frac{\sum_{0}^{n} \text { Pkt Recvd Time }- \text { Pkt Sent Time }}{n}$

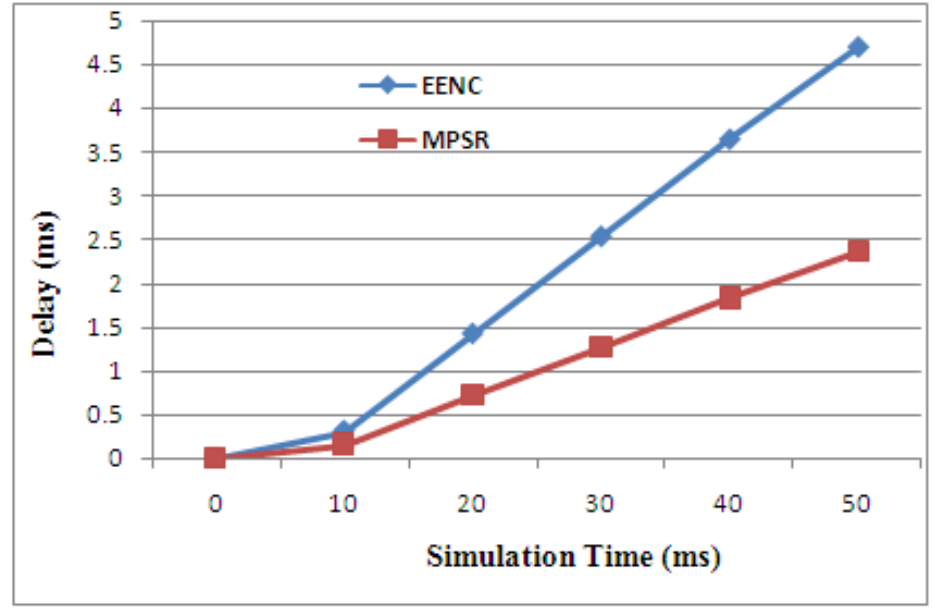

Figure.6 Average delay

Figure 6 shows that the delay value is low for the proposed scheme MEPSR than the existing scheme EENC. The minimum value of delay means that higher value of the throughput of the network. The delay in the network may lead to lose the information. The information must not get lost because of the delay factor, therefore proper sharing is required in this phenomenon during data transmission.

\subsection{THROUGHPUT:}

Throughput is the average of successful messages delivered to the base station. The throughput is estimated in using equation 6.

$$
\text { Throughput }=\frac{\sum_{0}^{n} \text { Pkts Received }(n) * \text { Pkt Size }}{1000}
$$


International Journal of Computer Networks \& Communications (IJCNC) Vol.9, No.6, November 2017

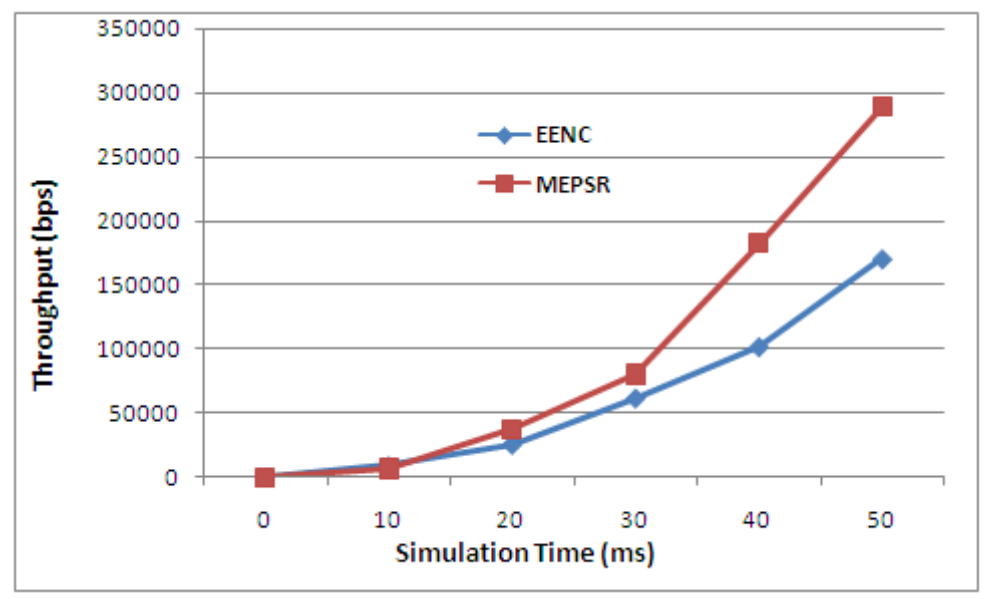

Figure.7 Throughput

Figure 7 shows that proposed scheme MEPSR has greater throughput when compared to the existing scheme EENC. Throughput is considered as the important parameter in determining the performance of the network. As long as there is improved throughput the system can perform in an efficient manner. The efficiency thus plays an important role in the communication network.

\subsection{RESIDUAL ENERGY:}

The persisting energy level in a node at the present time is called as RE. A measure of the RE gives the rate at which energy is utilized by the network performance. Figure 8 shows that the residual energy of the network is better for the proposed scheme MEPSR when compared with the existing scheme EENC. In MEPSR, reduce the node will be dead also As long as there is residual energy, the network will have an enhanced network lifetime in the network.

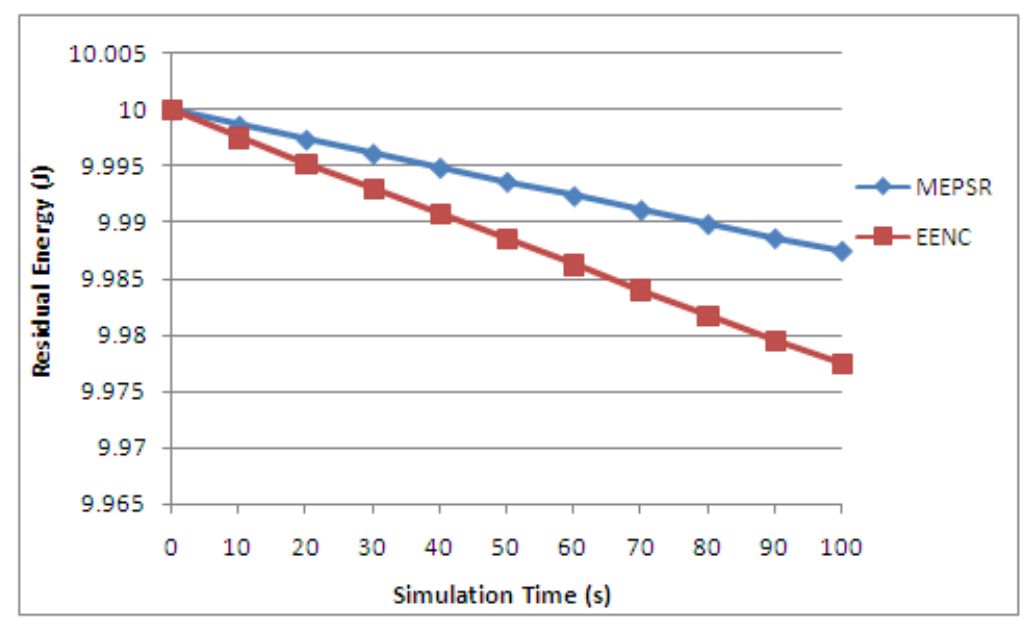

Figure.8 Residual Energy

\section{CONCLUSION}

This paper designs the Multicasting based Enhanced Proactive Sender Routing in MANETs. In this scheme, the source transmits the data to multiple destinations. This method uses the residual energy and transmission energy as most important parameters for multicasting in this 
communication network. Multicast is a very useful data transmission system for group-oriented applications. This algorithm explains an innovative multicasting algorithm that considers the transmission energy and residual energy while forwarding the data packets. Both theoretical and simulation outcomes demonstrate that MEPSR has better routing efficiency also improve the residual energy in the network. Simulation analysis is carried in this proposed system and it shows improved performance of throughput and reduces both the delay and energy consumption over the existing system.

\section{REFERENCES}

[1] Varaprasad, G., and Wahidabanu, R. S. D. (2013), "New power-aware multicast algorithm for mobile ad hoc networks", IEEE Potentials, Vol.32, No.2, pp.32-35.

[2] Suresh, H. N., Varaprasad, G., and Jayanthi, G. (2014), "Notice of Violation of IEEE Publication Principles Designing Energy Routing Protocol with Power Consumption Optimization in MANET", IEEE Transactions on Emerging topics in Computing, Vol.2, No.2, pp.192-197.

[3] Zhang, X. M., Wang, E. B., Xia, J. J., and Sung, D. K. (2013), “A neighbor coverage-based probabilistic rebroadcast for reducing routing overhead in mobile ad hoc networks", IEEE transactions on mobile computing, Vol.12, No.3, pp.424-433.

[4] Lin, K., Lai, C. F., Liu, X., \& Guan, X. (2012). Energy efficiency routing with node compromised resistance in wireless sensor networks. Mobile Networks and Applications, 17(1), 75-89.

[5] Ma, T., Liu, Y., and Zhang, Z. J. (2015), "An energy-efficient reliable trust-based data aggregation protocol for wireless sensor networks”, International Journal of Control Automation, Vol.8, No.3, pp.305-318.

[6] Das, S. K., and Tripathi, S. (2015), "Energy efficient routing protocol for MANET based on vague set measurement technique", Proceeding of Computer Science, Vol.58, pp.348-355.

[7] Gopinath, S., and Nagarajan, N. (2015), "Energy based reliable multicast routing protocol for packet forwarding in MANET", Journal of applied research and technology, Vol.13, No.3, pp.374-381.

[8] Ravi, R. R., and Jayanthi, V. (2015), "Energy efficient neighbor coverage protocol for reducing rebroadcast in MANET”, Proceeding of Computer Science, Vol.47, pp.417-423.

[9] Sandeep, J., and Kumar, J. S. (2015), "Efficient Packet Transmission and Energy Optimization in Military Operation Scenarios of MANET”, Proceeding of Computer Science, Vol.47, pp.400-407.

[10] Sara, Z., and Rachida, M. (2015), 'Energy-Efficient Inter-Domain Routing Protocol for MANETs', Proceeding of Computer Science, Vol.52, pp.1059-1064.

[11] Sumathi, K., and Priyadharshini, A. (2015), "Energy Optimization in Manets Using On-demand Routing Protocol”, Proceeding of Computer Science, Vol.47, pp.460-470.

[12] Pandey, S., and Mahapatra, R. P. (2015), "A centralized comparison of energy efficient routing protocol for mobile and static wireless sensor network", Proceeding of Computer Science, Vol. 48, 467-471.

[13] Arora, B. (2015), “An Adaptive Transmission Power Aware Multipath Routing Protocol for Mobile Ad hoc Networks", Proceeding of Computer Science, Vol.57, pp.1242-1248.

[14] Brar, G. S., Rani, S., Chopra, V., Malhotra, R., Song, H., and Ahmed, S. H. (2016), "Energy efficient direction-based PDORP routing protocol for WSN", IEEE Access, Vol.4, pp.3182-3194. 
International Journal of Computer Networks \& Communications (IJCNC) Vol.9, No.6, November 2017

[15] Gulati, M. K., and Kumar, K. (2015), "Stable Energy efficient QoS based Congestion and Delay aware Routing (SEQCDR) Protocol for MANETs", In Communications and Signal Processing (ICCSP), IEEE International Conference on, pp.0505-0511.

[16] Park, S. H. (2017), "Energy-Efficient Design of MIMO Processing for Two-Tier Wireless Sensor Networks", IEEE Wireless Communications Letters.

[17] Yadav, M. M., and Chavan, G. T. (2013), "Predictive Location-Based QoS Routing with Admission Control in Mobile Ad-hoc Networks to improve QoS. Extraction”, Vol.2, No.7.

[18] Yakine, F., znd Idrissi, A. (2015), "Energy-aware topology control and QoS routing in ad-hoc networks", Proceeding of Computer Science, Vol.56, pp.309-316.

[19] Srivastava, P., and Kumar, R. (2015), "A novel multi metric QoS routing protocol for MANET", In Advances in Computing, Communications and Informatics (ICACCI), IEEE International Conference on, pp. 1746-1752.

[20] Surendran, S., and Prakash, S. (2015), “An ACO look-ahead approach to QOS enabled fault-tolerant routing in MANETs", China Communications, Vol.12, No.8, pp.93-110.

[21] Rao, A. R., Kumari, V. V., and Reddy, C. S. (2016), "Backup route establishment for QoS routing protocol in MANET", In Signal Processing, Communication, Power and Embedded System (SCOPES), IEEE International Conference on, pp. 856-862. 